\title{
Norois
}

Environnement, aménagement, société

\section{Veschambre (V.) - Traces et mémoires urbaines, enjeux sociaux de la patrimonialisation et de la destruction}

Rennes, PUR, 2008, 315 p.

Sébastien Jacquot

\section{CpenEdition}

Journals

Édition électronique

URL : http://journals.openedition.org/norois/2662

DOI : $10.4000 /$ norois. 2662

ISBN : 978-2-7535-1557-4

ISSN : $1760-8546$

Éditeur

Presses universitaires de Rennes

Édition imprimée

Date de publication : 15 décembre 2008

Pagination : 181-183

ISBN : 978-2-7535-0805-7

ISSN : 0029-182X

Référence électronique

Sébastien Jacquot, «Veschambre (V.) - Traces et mémoires urbaines, enjeux sociaux de la patrimonialisation et de la destruction », Norois [En ligne], 209 | 2008/4, mis en ligne le 01 mars 2009, consulté le 21 septembre 2020. URL : http://journals.openedition.org/norois/2662 ; DOI : https:// doi.org/10.4000/norois.2662 
Les guerres maritimes avec les Anglais au XVIII e siècle et le rôle joué par les Vendéens, officiers de marine, négociants et marins, montrent le déclin des activités et un repli que les événements de la Révolution ne font que confirmer. Jacques Péret, Bernard Michon et Philippe Bossis nous donnent de précieuses études sur ce thème, au travers de portraits d'acteurs, que ce soit Collinet ou Serventeau et autres armateurs et négociants sablais en particulier. Les difficultés du port et de ses activités se mesurent par la baisse de population, la ville passe de 10000 à 5000 habitants!

Les études sur la période révolutionnaire remettent en cause l'analyse des comportements des populations. On considérait jusqu'alors ces dernières comme plus favorables aux idées révolutionnaires que l'intérieur du département. Les contributions nuancent cet a priori. Il n'en demeure pas moins que la ville des Sables-d'Olonne restera fidèle à la République et résistera au siège des insurgés.

Les premières conserveries s'installent sur le littoral vendéen à partir de 1850. Elles connaissent un grand succès et en 1880 on en compte 25, et 28 en 1913, avec près de 4000 ouvriers, surtout des femmes. L'origine des capitaux, les liens avec les armateurs locaux, les avatars des entreprises, les difficultés nées des apports irréguliers, nous sont présentés par Xavier Dubois dans une contribution très documentée. De même le rôle des chantiers sablais est rappelé, notamment avec les pêcheurs de l'île de Groix.

Enfin plusieurs contributions font le point des évolutions récentes du tourisme littoral et de ses conséquences multiples sur les transformations des sociétés locales, ainsi que des difficultés présentes de la pêche. On notera également une contribution du recteur Alain Miossec quant aux dangers de l'érosion sur les côtes vendéennes et les solutions apportées par différentes techniques dont il a été un observateur attentif.

On regrettera l'absence d'une analyse socio-politique des comportements contemporains des populations. Il n'y a pas non plus de description de la mise en place du tou-

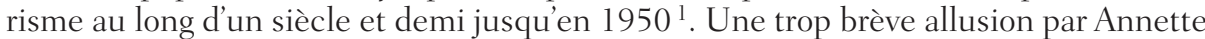
Roux, PDG des chantiers Beneteau, des activités liées à la plaisance n'épuise pas le sujet (chantiers, ports, écoles de voile et courses au large), alors même que le Vendée Globe est devenu un fait emblématique de la Vendée qui gagne, au même titre que la grande machinerie du Puy du Fou.

Jean RENARD

\section{VESCHAMBRE (Vincent), 2008. - Traces et mémoires urbaines, enjeux sociaux de la patrimonialisation et de la destruction, Rennes, PUR, $315 \mathrm{p}$.}

Comment les enjeux mémoriels se traduisent-ils au sein de l'espace urbain? Que révèlent-ils des processus d'appropriation de l'espace et des luttes pour la légitimation? Comment penser les mémoires éclatées et concurrentes, dominantes ou marginales? L'ouvrage Traces et mémoires urbaines, enjeux sociaux de la patrimonialisation et de la destruction, issu de l'HDR de Vincent Veschambre, approfondit ce questionnement entre inégalités sociales et productions mémorielles. Les processus mémoriels sont analysés dans le cadre d'une géographie sociale, attentive aux « enjeux de distinction, de marquage et d'appropriation de l'espace par certains groupes, certains pouvoirs »

1. Il est vrai que l'ouvrage récent de Johan Vincent : L'intrusion balnéaire : les populations littorales bretonnes et vendéennes face au tourisme (1800-1945), paru aux PUR en 2007, est une excellente analyse de la mise en tourisme de ces littoraux et des conséquences sur les sociétés locales, tout autant complices et bénéficiaires que victimes de cette intrusion. 


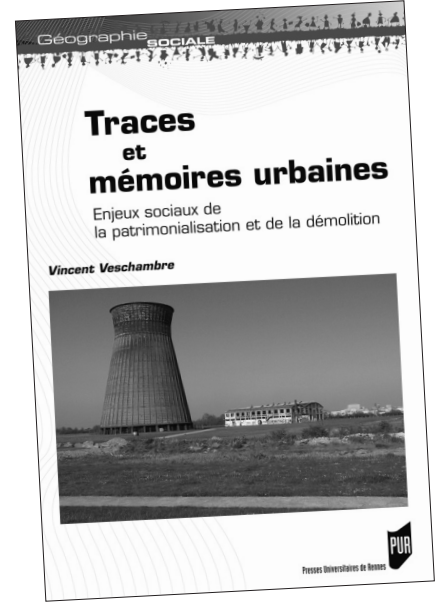

(p. 285), empruntant notions et questionnements à la sociologie de P. Bourdieu. Le fil conducteur de cet ouvrage se situe dans l'articulation entre traces, espace et pouvoir, sur les versants matériels et symboliques.

Les terrains et études qui alimentent la réflexion sont variés : Angers (gentrification de la Doutre, destructions des grands ensembles, lieux de commémoration de ATD Quart-monde) et son agglomération (patrimoine industriel entre destruction et valorisation à Angers et Trélazé), le camp de Montreuil Bellay, Firminy le Vert, Caen, Le Mans, Nantes, et sont accompagnés de lieux plus lointains fonctionnant de façon paradigmatique (destructions en Yougoslavie, Berlin et Bucarest, camps de Belzec et Auschwitz-Birkenau). Cette pluralité des terrains nous rapproche d'une géographie générale, qui construit concepts et résultats à partir d'études variées, avec une dimension anthropologique affirmée.

Les notions nécessaires à l'exposition des résultats des recherches sont posées en début d'ouvrage. Le couple trace/marque constitue le matériau premier. Les traces sont les vestiges et indices du passé, qui subsistent de façon non intentionnelle. Elles peuvent être l'objet d'une réactivation contemporaine (patrimonialisation) ou au contraire d'un effacement (destruction). Les marques renvoient à une action intentionnelle, le marquage, fonctionnant comme signature d'un acteur social dans un lieu. La réactivation d'une trace (patrimonialisation) opère comme un marquage social. Traces et marques constituent les supports matériels de l'appropriation spatiale, pour la légitimer, la revendiquer ou la contester. Sur le versant symbolique traces et marques constituent des mémoires urbaines permettant de (dé-)légitimer des appropriations particulières. V. Veschambre montre que ces mémoires sont plurielles, éventuellement mises en scène (commémorations), étant prises dans les «stratégies symboliques » de lutte pour l'inscription dans l'espace et le temps et donc la légitimation de sa présence dans un lieu.

L'ouvrage est organisé en trois parties, chacune consacrée à une facette de l'inscription mémorielle en lien avec l'appropriation de l'espace : la patrimonialisation, la destruction et la mise en mémoire.

La première partie, consacrée au processus de patrimonialisation, propose une critique de quelques lieux communs. Le discours aux connotations malthusiennes (p. 52) fustigeant l'inflation des catégories et lieux patrimoniaux est interprété comme une volonté de limiter l'accès au marquage patrimonial. En effet, incorporer de nouveaux patrimoines menace la valeur des patrimoines établis, et partant la position symbolique de ses détenteurs. De même, la conception holiste du patrimoine, qui postule une identification consensuelle, masque les enjeux de légitimation d'un ordre social. Ainsi l'auteur met en évidence la dimension idéologique du patrimoine. La patrimonialisation constitue un enjeu social et symbolique, étant « un des attributs de la position sociale des individus et des groupes et l'un des paramètres des inégalités sociales ». L'émergence de nouveaux patrimoines permet « pour des catégories sociales qui n'ont pas hérité de patrimoines auxquels s'identifier (châteaux, hôtels, monuments liés au pouvoir...) de se forger un patrimoine en propre, une visibilité, une légitimité » (p. 39). Cet enjeu identitaire et symbolique révèle les liens entre patrimonialisation et appropriation et contrôle de l'espace, le patrimoine conférant « un droit de présence et une légitimité dans la ville» (p. 73), comme le montrent les liens intrinsèques entre la patrimonialisation et la gentrification. 
L'envers de la patrimonialisation, la démolition, est étudiée dans la seconde partie à partir des mêmes notions de traces, marques et des luttes pour l'appropriation de l'espace, ce qui confère en dépit de terrains et lieux différents une unité théorique au propos. La dimension idéologique de la démolition apparaît de façon crue dans les destructions menées en Afghanistan ou à Bucarest. Ces exemples paradigmatiques permettent de définir la démolition comme un effacement de marques par un pouvoir désirant instaurer un nouvel ordre, et d'illustrer la violence symbolique présente dans toute démolition, y compris celles pouvant apparaître comme plus banales : grands ensembles et traces industrielles. La destruction de traces industrielles peut s'apparenter à une « invisibilisation » (p. 175) de la classe ouvrière et celle des grands ensembles à un rejet d'une conception du logement social. Ces deux modes de la démolition constituent un envers de l'appropriation de l'espace, appelé « expropriation de l'espace ».

La troisième partie opère une radicalisation du questionnement puisque sont envisagés les modes d'actualisation mémorielle des traces effacées, à travers la production de monuments et la commémoration. L'analyse de la mise en mémoire des camps d'extermination de Belzec et Auschwitz-Birkenau ou d'enfermement des gitans de Montreuil Bellay fait apparaître la mise en mémoire comme recours contre l'oubli et la volonté d'effacement des traces. V. Veschambre est attentif aux différents registres de cette mise en mémoire, de la production de monuments (renvoyant à différentes constructions d'ensembles de victimes) à l'anti-monument, où « l'espace tout entier est investi de la fonction mémorielle » (p. 209). Toutefois la mise en mémoire n'est pas uniforme et relève également d'un travail de visibilisation et de légitimation, posant la question de la production de mémoires des dominés. L'expérience menée par l'auteur avec ATD Quart-monde permet d'illustrer les difficultés d'une telle entreprise : produire des marques ou réinvestir des traces, occuper un haut lieu central ou un lieu significatif de la misère? Le cas de la mémoire ouvrière montre également le décalage qui peut s'instaurer entre mémoire ouvrière et conservation de traces décontextualisées et réinterprétées par d'autres. La mise en mémoire, dont la dimension spatiale est démontrée, est prise dans les enjeux d'appropriation de l'espace et de légitimation.

Ce cheminement entre patrimoine, destructions et mémoires permet de proposer en conclusion des pistes convaincantes sur la notion de lieu et du passage de l'espace au lieu, perçu comme le résultat d'un marquage (et donc d'une construction sociale) conférant des significations. Au final cet ouvrage, à partir d'une géographie sociale de la patrimonialisation et de la mise en mémoire, fait apparaître les liens entre légitimation et appropriation spatiale, prenant appui sur les traces et marques prises dans la dynamique des différenciations socio-spatiales et luttes pour la reconnaissance.

Sébastien JACQUOT 